\title{
OSTEOPATIA HIPERTRÓFICA PULMONAR- ALTERAÇÕES CLÍNICAS E RADIOGRÁFICAS EM UM PACIENTE CANINO
}

\author{
Larissa Correa Hermeto1, Suelen Sâmera Pereira Fernandes², Paulo Henrique de \\ Affonseca Jardim³ ${ }^{3}$, Douglas Rodrigo Mattei ${ }^{4}$, Thais Pelisari² \\ 1 UNESP - Campus Jaboticabal \\ 2 FAD \\ 3 UFMS \\ ${ }^{4}$ UFMT \\ Correspondência: Larissa Correa Hermeto: Iarissa_hermeto@yahoo.com.br
}

RESUMO: A osteopatia hipertrófica é uma doença incomum que tem sido descrita em cães frequentemente em associação a processos neoplásicos pulmonares primários ou metastáticos. Uma cadela sem raça definida com dez anos de idade foi atendida no hospital veterinário de Dourados-MS, por apresentar prostração, dificuldade de locomoção, aumento de volume edematoso em todos os membros. O exame radiográfico revelou aumento de volume de tecidos moles e reação periosteal de aspecto paliçada no rádio, ulna, metacarpos. Também foi detectada massa tumoral difusa em parênquima pulmonar. O diagnóstico final foi de osteopatia hipertrófica pulmonar associada à metástase pulmonar de adenocarcinoma.

Palavras-chave: cão; neoplasia pulmonar; reação perisoteal

\section{HYPERTROPHIC PULMONARY OSTEOPATHY- CLINICAL AND RADIOGRAPHIC ALTERATIONS IN A CANINE PATIENT}

\begin{abstract}
Hypertrophic osteopathy is a rare disease which has been reported in dogs often associated with primary or metastatic pulmonary neoplastic processes. A ten-year-old female mongrel was admitted to the veterinary hospital in the city of Dourados-MS for presenting prostration, limited mobility, and edematous swelling of all the limbs. The radiographic examination revealed soft tissues swelling and lattice-shaped periosteal reaction in the radius, ulna, and metacarpals. A diffuse tumor mass in the pulmonary parenchyma was also detected. The final diagnosis was hypertrophic osteopathy associated with metastatic lung adenocarcinoma.
\end{abstract}

Key Words: dog; lung neoplasma; periosteal reaction 


\section{INTRODUÇÃO}

A osteopatia hipertrófica (OHP) é uma rara desordem osteoproliferativa periosteal generalizada que afeta principalmente as extremidades dos ossos longos (Alan, 2007), gerada por uma resposta óssea a uma doença crônica, geralmente intratorácica (Ogilvie, 2001).

É uma reação periosteal difusa que irá resultar em formação de tecido ósseo novo ao redor dos ossos longos, metatársicos e metacárpicos (Johnson e Hulse, 2005).

A fisiopatologia da doença ainda não está bem esclarecida, mas várias teorias ainda não comprovadas tentam explicar a origem da patologia que é desconhecida. Alguns estudos relatam que o pulmão deixa de sintetizar uma substância vasodilatadora e remodeladora de tecido ósseo, onde o fator de crescimento pode ser proveniente de plaquetas (Woodard, 2000). Outra teoria consiste de um reflexo neuro-vagal que promove vasodilatação periférica, sendo uma resposta de neoplasia primária (Filgueiras et al., 2002). Postulou-se que as lesões pulmonares levam a alterações vasomotoras reflexas (mediadas pelo nervo vago) e a um aumento no fluxo sanguíneo para as extremidades (Weisbrode, 2009). O aumento do fluxo sangüíneo seria secundário à estimulação de trajetos neurais aferentes (Manley, 1995). Outras teorias citam fatores humorais, hipóxia ou combinação de ambos (Susaneck e Macy, 1982).

A causa da doença pode ser uma síndrome neoplásica (incluindo tumores primários ou metastáticos, carcinoma esofágico, rabdomiossarcoma da vesícula urinária, carcinoma de células transicionais renais e nefroblastoma) ou estar associada a outras patologias (lesões granulomatosas, megaesôfago crônico, ducto arterioso patente, endocardite bacteriana e dirofilariose) (Anderson et al., 2004), sendo a causa mais comum a neoplasia pulmonar, porém, também são relatados casos da doença em animais com neoplasias primarias em cavidade abdominal (Becker, 1999). Quando em cavidade abdominal, a neoplasia geralmente esta associada à vesícula urinária, fígado e ovário (Filgueiras et al., 2002).

Os sinais clínicos principais são letargia, relutância ao mover-se e edema nas extremidades distais, e geralmente os membros afetados estão quentes (Schulz, 2008).

O diagnóstico é realizado com facilidade, devido à apresentação radiográfica característica das alterações (Costa et al., 2008). Exames laboratoriais podem não ser esclarecedores para o diagnóstico da OHP, porém, sabe-se que a atividade dos osteoblastos em depositar tecido ósseo eleva os valores séricos de fosfatase alcalina (Bush, 2004).

O tratamento consiste na remoção da causa primária, o que resulta na regressão dos sinais clínicos, assim como parcial ou quase total das lesões periosteais, como citado em casos de procedimentos cirúrgicos terapêuticos como: pneumonectomia ou lobectomia pulmonar para remoção de corpo estranho em brônquio (Caywood et al., 1985), osteossarcoma primário (Brodey, 1971; Fox et al., 1994), carcinoma adenoescamoso (Lee et al., 2012) fibrossarcoma primário (Madewell et al., 1978) e adenocarcinoma (Kelly, 1984); toracotomia, vagotomia unilateral e remoção de massa tumoral em pulmão (Watson e Porges, 1973); excisão de massa tumoral intratorácica conectada ao tronco vagal (Hara et al., 1995); e excisão de neoplasia pulmonar (Brodey, 1971). 


\section{RELATO DE CASO}

Uma cadela, SRD, idade de 10 anos, pesando $20 \mathrm{~kg}$ foi atendida no Hospital Veterinário de Dourados-MS, e segundo 0 proprietário há aproximadamente dois meses apresentava edema nos membros, e há quinze dias não estava caminhando. Outras observações feitas pelo proprietário foram presença de secreção ocular e nasal que apresentava aspecto purulento, perda de peso progressiva, anorexia, prostração, apatia, dispnéia, além de edema na região da vulva e presença de ectoparasitas. Uma informação adicional referente ao histórico do animal, é que sete meses antes do início deste quadro, o mesmo apresentou um nódulo em uma das mamas, no qual foi realizado mastectomia regional nesta época.

Ao exame físico, detectou-se que as mucosas orais e oculares apresentavam-se hipocoradas, a ausculta pulmonar demonstrou estertores úmidos e frequência respiratória de 48 movimentos por minuto, acompanhada de moderada dispnéia expiratória e submacicez torácica a percussão. Observou-se ainda moderada desidratação, discreta hipotermia $\left(37,9^{\circ} \mathrm{C}\right)$, tempo de preenchimento capilar em dois segundos, e frequência cardíaca em 136 batimentos por minuto. $\mathrm{Na}$ inspeção corpórea do animal observou-se edema generalizado de membros com sinal de Godet positivo em algumas regiões dos membros, (figura 1) e em outras consistência firme e doloroso a palpação, sendo apresentavam-se extremamente pesados, algumas escoriações pelo corpo, presença de secreção mucopurulenta nas narinas, vulva edemaciada associada a secreção purulenta.

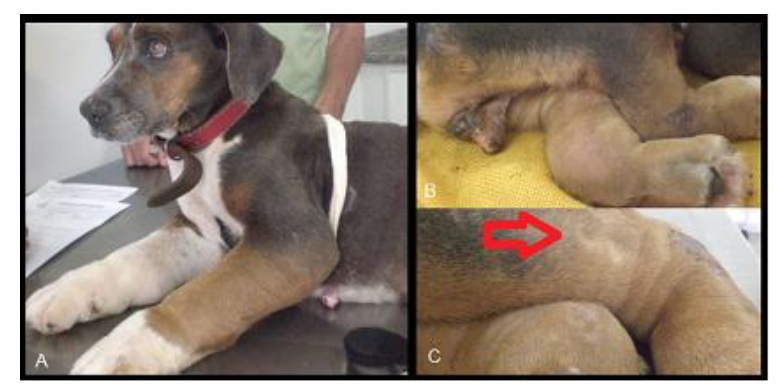

Figura 1: A- Edema de membros anteriores. B- Edema de membros posteriores e algumas escoriações cutâneas. C- Sinal de Godet positivo (seta).

Após o exame físico o animal foi submetido à medicação analgésica com butorfanol na dose de $0,4 \mathrm{mg} / \mathrm{kg}$ por via intramuscular para a realização de exames complementares pois o animal apresentava extremo desconforto a qualquer manipulação. Os exames solicitados foram: radiografia de membros e tórax, ultrassonografia abdominal, e exames laboratoriais. $\mathrm{Na}$ imagem radiográfica do membro anterior esquerdo no posicionamento dorso palmar, foram observados os achados radiográficos (figura 2): presença de proliferação óssea do tipo "paliçada" (seta azul) em diáfise de ossos metacarpianos, falanges proximais e intermediarias, e de radio e ulna além do acentuado aumento de volume dos tecidos mole na região avaliada devido o edema (seta vermelha).

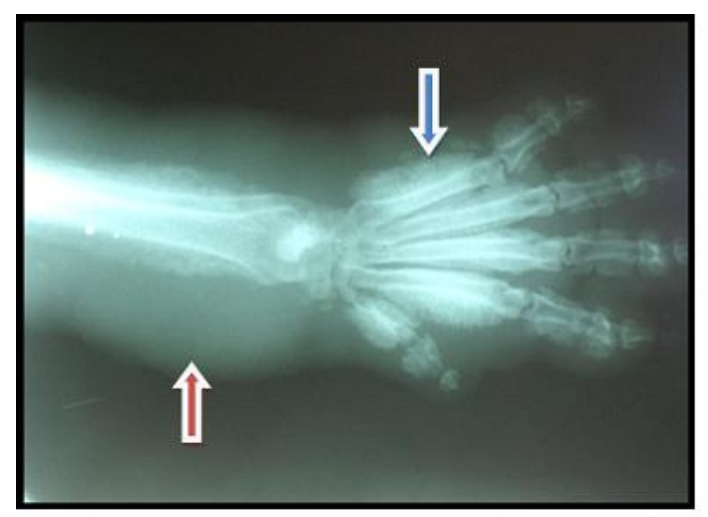

Figura 2: Imagem radiográfica exibindo proliferação óssea do tipo "paliçada" (seta azul) e acentuado aumento de volume dos tecidos mole (seta vermelha).

$\mathrm{Na}$ radiografia torácica foi evidenciado incremento de radiopacidade difuso, com imagem sugestiva de múltiplos nódulos em toda a extensão dos campos pulmonares. 
As alterações radiográficas, conjuntamente com os achados clínicos levam a possibilidade de diagnóstico como osteopatia hipertrófica pulmonar.

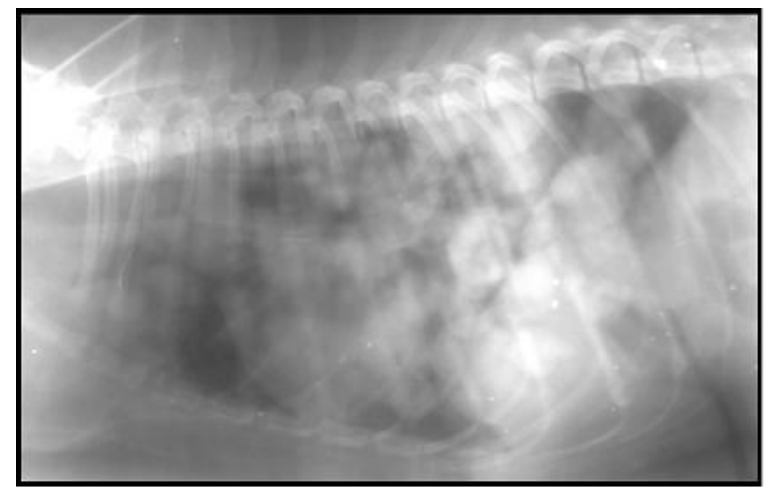

Figura 3: Radiografia torácica lateral evidenciando inúmeras imagens radiopacas nodulares.

Após a conclusão radiográfica, foi explanado ao proprietário as possibilidades terapêuticas como quimioterapia e terapia paliativa, mas devido ao estado crítico do paciente, o qual apresentava desconforto e disseminação da doença e associado infelizmente a falta de recursos financeiros do proprietário, o mesmo decidiu pela realização da eutanásia no animal para abreviar o sofrimento do mesmo. Os exames laboratoriais que seriam solicitados foram cancelados. $O$ laudo histológico das neoformações torácicas concluiu adenocarcinoma.

\section{DISCUSSÃO}

Segundo Filgueiras et al., (2002), a OHP é uma rara patologia que caracteriza-se pela neoformação óssea periosteal, que dificulta a locomoção e gera intensa sensibilidade dolorosa ao animal. Os cães acometidos apresentam inquietação, relutância em movimentar-se e aumento de volume da parte distal dos membros (Becker, 1999). Em um levantamento de achados clínico- patológicos em sete cães acometidos pela OHP realizado por Trost et al. (2012), as principais queixas relacionadas às alterações ósseas foram: limitação nos movimentos dos membros, dor à palpação, aumento de volume e claudicação, sinais clínicos também evidenciados no paciente deste relato.

A idade da paciente corroborou com os dados encontrados na literatura, pois por estar associada a neoplasias, a OHP ocorre principalmente em animais idosos (Kealy e Mcallister, 2000), e de acordo com Blassioli et al. (2007) fêmeas são abordadas principalmente devido a casos de neoplasias mamárias.

$\mathrm{O}$ achado patológico mais comum nos animais afetados é o aumento do fluxo sanguíneo para as extremidades, resultando no supercrescimento do tecido conectivo, com consequente metaplasia fibrocondróide e neoformação óssea subperiosteal (Alan, 2007), o que foi diagnosticado clinicamente na presença de edema de membros generalizada e pelas alterações radiográficas encontradas.

Foi diagnosticado como possível causa primária o tumor mamário que 0 animal apresentou em seu histórico, e que desencadeou 0 processo metastático pulmonar, e segundo levantamento de Johnson e Watson (2004), de 180 casos em cães, 98\% tinham doenças intratorácicas e 92\% apresentavam neoplasia metastática pulmonar ou tumores primários do pulmão ou do esôfago torácico, alguns apresentaram pneumonia, endocardite ou diofilariose. Trost et al. (2012) apontaram em levantamento, que todos os casos avaliados apresentaram neoplasias pulmonares, mas que apenas 1 dos 7 casos avaliados apresentou neoplasia pulmonar primária sendo que os outros seis casos apresentaram metástase pulmonar de tumores primários não torácicos. Para Bettini et al. (2009), a OHP pode ser uma síndrome paraneoplásica e é mais frequentemente associada a tumores pulmonares. Corroborando com a literatura, foi diagnosticado 
adenocarcinoma pulmonar, sendo para Sato et al. (2005) este tipo histológico o mais comum encontrado em tumores pulmonares no cão.

Para Kealy e MCallister (2000), o sucesso do tratamento da doença pulmonar é o suficiente para a regressão das alterações periosteais, mas Ogilvie (2001) sugere outros tratamentos, como vagotomia unilateral no lado da lesão pulmonar, incisão através da pleura parietal, ressecção subperiosteal da costela, vagotomia cervical bilateral e utilização de analgésicos. Em muitos casos como de Filgueiras et al. (2002), infelizmente a eutanásia é sugerida devido à intensa dificuldade respiratória, aos achados radiográficos e à extensão da patologia encontrada. No animal deste relato não seria possível a terapia cirúrgica da enfermidade pulmonar, pois a disseminação da neoplasia era intensa e o estado crítico da paciente levou a opção de sugerir um tratamento paliativo associado à quimioterapia ou a eutanásia, o qual foi optado pelo proprietário pela eutanásia.

\section{CONCLUSÃO}

No presente estudo, as alterações clínicas e radiográficas encontradas foram semelhantes ao que se encontra na literatura descrita, mas devido ao avanço da enfermidade não foi possível a instituição de uma terapêutica.

\section{REFERÊNCIAS}

ALAN, G. S., Radiographic signs of joint disease in dog and cats In: THRALL, D. E. Textbook of Veterinary Diagnostic Radiology. 5 ed.

Editora: Saunders Elsevier. St. Louis - Missouri, 2007.

ANDERSON, T.P.; WALKER, M.C.; GORING, R.L. Cardiogenic hypertrophic osteopathy in a dog with right-to-left shunting patent ductus arteriosus. Journal of the American Veterinary Medical Association, v.224, p.1464, 2004.
BECKER, T. J.; PERRY, R. L.; WATSON, G. L. Regression of hypertrophic osteopathy in a cat after surgical excision of an adrenocortical carcinoma. Journal of the American Animal Hospital Association. v.35, n.6, p.499-505, 1999.

BETTINI, G; MARCONATO, L; MORINI, M. et al. Thyroid transcription factor-1

immunohistochemistry: diagnostic tool and malignancy marker in canine malignant lung tumours. Veterinary and Comparative.

Oncology, v.7, p.28-37, 2009.

BLASSIOLI, M.S; FERRARO, C.M; M, KOLBER. Osteopatia hipertrófica pulmonar relato de caso. In: $34^{\circ}$ Congresso Brasileiro de Medicina Veterinária, Anais. Santos/SP, 2007.

BRODEY, R.S. Hypertrophic osteoarthropathy in the dog: a clinicopathologic survey of 60 cases. Journal of the American Veterinary Medical Association, v.159, n.10, p.1242-1256, 1971.

BUSH, B. M. Interpretação de Resultados Laboratoriais Para Clínicos de Pequenos Animais. São Paulo: Roca, 2004, p.96-97.

CAYWOOD, D.D.; KRAMEK, B.A.; FEENEY, D.A. et al. Hypertrophic osteopathy associated with a bronchial foreign body and lobar pneumonia in a dog. Journal of the American Veterinary Medical Association, v.186, n.7, p.698-700, 1985.

COSTA, F. S.; MACHADO, F. M.; PEREIRA, B. J. et al. Osteopatia hipertrófica - relato em cão jovem. Publicações em Medicina Veterinária e Zootecnia,. v.2, n.17, 2008.

FILGUEIRAS, R. R.; SILVA, J. C. P.; ILÓRIA, M. I. V. et al. Osteopatia hipertrófica em cão relato de caso. Clínica Veterinária, v.7, n.36, p.28-32, 2002.

FOX, L.E.; KING, R.R.; MAYST, M.C. et al. Primary pulmonary osteosarcoma in a dog. Journal of Small Animal Practice, v.35, p.329332, 1994.

HARA, Y.; TAGAWA, M.; EJIMA, H. et al. Regression of hypertrophic osteopathy following removal of intrathoracic neoplasia derived from vagus nervus in a dog. Journal of Veterinary Medical Science, v.57, n.1, p.133-135, 1995.

JOHNSON, K.A; WATSON, A.D.J. Doenças Esqueléticas. In ETTINGER, Stephen J.; FELDMAN,Edward C. Tratado de Medicina Interna Veterinária. $5^{\underline{a}}$ ed. v.2, Rio de Janeiro, 
RJ: Guanabara Koogan S.A, 2004. P-13041309, 2004.

JOHNSON, A.L; HULSE, D.A. Outras Osteopatias e Artropatias. In: FOSSUM, T.W. HEDLUND,C.S.; HULSE, D.A.; JOHNSON,A.L; SEIM,H.B; WILLARD,M.D.; CARROLL,G.L. Cirurgia de pequenos animais. $2^{a}$ ed. São Paulo, SP: Rocca, 2005, p. 1160-1161.

KELLY, M.J. Long-term survival of a case of hypertrophic osteopathy with regression of bony changes. Journal of the American Animal Hospital Association, v.20, n.3, p.439-444, 1984.

KEALY, J. K.; MCALLISTER, H. Ossos e articulações In:Radiografia e Ultra-sonografia do Cão e do Gato. $3^{a}$ ed. Manole: São Paulo, 2005. p.253 - 338 .

LEE, JH.; YOON, HY.; KIM, NH. et al. Hypertrophic osteopathy associated with pulmonary adenosquamous carcinoma in a dog. The Journal of Veterinary Medical Science, v.74, n.5, p.667-672. 2012.

MADEWELL, B.R.; NYLAND, T.G.; WEIGEL, J.E. Regression of hypertrophic osteopathy following pneumonectomy in a dog. Journal of the American Veterinary Medical Association, v.172, n.7, p.818-821, 1978

MANLEY, P. Diseases affecting bone. In: OLMSTEAD, M.L. Small animal orthopedics. St. Louis: Mosby, 1995.Cap. 20, p.427-435.

OGILVIE, G.K. Paraneoplastic Syndromes In: WITHROW, S.J.; VAIL, D.M. Small Animal Clinical Oncology. 4 ed. Philadelphia: Saunders Elsevier, 2001, p.303-310.

SATO, T.; ITO, J.; SHIBUYA, H.; ASANO, K.; WATARI, T. Pulmonary adenosquamous carcinoma in a dog. Journal of the American. Veterinary Medical Association v.52, p.510513, 2005.

SCHULZ, K. Outras doenças dos ossos e articulações, In: Fossum, T.W.; Cirurgia de Pequenos Animais, 3 ed, Ed. Mosby elsevier, 2008, p. 1333-1334

SUSANECK, S.J.; MACY, D.W. Hypertrophic osteopathy. Compendium on Continuing Education for the Practicing Veterinarian, v.4, n.8, p.689-693, 1982.

TROST, M.E.; KOMMERS, G.D.; SILVA, T.M. et al. Osteopatia hipertrófica em sete cães.
Pesquisa Veterinária Brasileira, v.32, n.5, p.424-429, 2012.

WATSON, A.D.J.; PORGES, W.L. Regression of hypertrophic osteopathy in a dog following unilateral intrathoracic vagotomy. Veterinary Record, v.93, n.9, p.240-243,1973.

WEISBRODE, A.E. Ossos e articulações, In: Bases da patologia em Veterinária. McGAVIN, M.D.; ZACHARY, J.F. 4 ed. 2009. Ed. Mosby Elsevier, p.1084.

WOODARD, J. C. Sistema esquelético In: Patologia Veterinária. JONES, T. C., HUNT, R. D., KING, N. M.. 6ํe ed. Manole: São Paulo, 2000. p.913-961. 